\title{
LBP-guided Active Contours
}

\author{
Michalis A. Savelonas, Dimitris K. lakovidis, and Dimitris Maroulis \\ Department of Informatics and Telecommunications, University of Athens, \\ Panepistimiopolis, Illisia, GR-15784 Athens, Greece \\ \{m.savelonas, d.iakovidis, d.maroulis\}@di.uoa.gr
}

\begin{abstract}
This paper investigates novel LBP-guided active contour approaches to texture segmentation. The Local Binary Pattern (LBP) operator is well suited for texture representation, combining efficiency and effectiveness for a variety of applications. In this light, two LBP-guided active contours have been formulated, namely the scalar-LBP Active Contour (s-LAC) and the vector-LBP Active Contour ( $v$-LAC). These active contours combine the advantages of both the LBP texture representation and the vector-valued Active Contour Without Edges model, and result in high quality texture segmentation. $s$-LAC avoids the iterative calculation of active contour equation terms derived from textural feature vectors and enables efficient, high quality texture segmentation. $v$ LAC evolves utilizing regional information encoded by means of LBP feature vectors. It involves more complex computations than $s$-LAC but it can achieve higher segmentation quality. The computational cost involved in the application of $v$-LAC can be reduced if it is preceded by the application of $s$-LAC. The experimental evaluation of the proposed approaches demonstrates their segmentation performance on a variety of standard images of natural textures and scenes.
\end{abstract}

Keywords: Local Binary Patterns, Texture Segmentation, Active Contours.

\section{Introduction}

Texture segmentation methods based on active contour approaches have received considerable attention over the past few years (Theodoridis and Koutroumbas, 2006; Paragios and Deriche, 1999; Lehmann et al, 2001; Sandberg et al, 2002; Aujol et al, 2003; Rousson et al, 2003; Sagiv et al, 2004; Huang et al, 2004; He et al, 2004; Allili et al, 2004; Pujol and Radeva, 2004; Lee et al, 2005), by exploiting advances in the active contour research such as contour smoothness, noise robustness and topological adaptability. This emerging trend in the area of texture segmentation has been reinforced by the vector formulation of recent active contour approaches (Chan et al, 2002; Sandberg and Chan, 2005) introduced to provide a natural platform for the embedment of textural features. Such methods constitute an essential first step in computer vision applications, which are as diverse as medical image analysis, industrial monitoring of product quality, content-based image retrieval and remote sensing.

The main notion of the active contour approach to texture segmentation relies on the deformation of initial contours towards the boundaries of image regions to be segmented. The deformation is realized by minimizing an energy functional, designed so that its local minimum is reached at target boundaries. Active contour models lead to 
continuous, closed or open, curves without requiring edge-linking operations. The original active contour approach (Kass et al, 1988) is boundary-based utilizing intensity gradients to guide contour evolution. However, in the case of objects whose boundaries are either smooth or not necessarily defined by gradient, such methods may result in boundary "leakage" (Chan and Vese, 2001). Moreover, the parametric formulation of the original active contour approach does not allow for changes in the topology of the evolving contour, such as splitting or merging.

Alternative active contour approaches have been proposed to surpass the aforementioned limitations: Caselles et al, (1997) introduced the Geodesic Active Contour (GAC) model which uses the level set method, originally proposed in (Osher and Sethian, 1988), in order to facilitate topological adaptability. However, the GAC model inherits the dependency on gradients of the original active contour approach and thus cannot prevent boundary "leakage" (Suri et al, 2002). Chan and Vese, (2001) proposed the Active Contour Without Edges (ACWE) model which is a level set and region-based active contour model, following the Mumford-Shah segmentation approach (Mumford and Shah, 1989). The ACWE model deals with the problem of boundary "leakage" by utilizing intensity integrals calculated over the regions inside and outside the contour. This model was later extended and then generalized by Chan et al, (2002) and Sandberg and Chan, (2005) respectively, for vector-valued images by replacing the scalar gray-level intensities with vectors of color channel intensities to guide contour evolution. However, the information derived from intensity integral operations can be misleading for texture segmentation tasks as regions of different textures may have equal average intensities. Therefore the utilization of ACWE based on image intensities can be considered unsuitable for texture segmentation, either in its original or in its generalized form. However, its region-based formulation could be exploited for capturing textural information, derived from features not necessarily exhibiting high gradients at object boundaries.

Latest advances in active contour research focus on the incorporation of textural features to guide the contour evolution. The methods that have been proposed span two categories:

1) Gabor and wavelet-based methods. Gabor-based methods involve transformation of input images into different scales, frequencies and orientations by Gabor filtering. The associated filter-bank responses are used to generate textural feature vectors to guide the contour evolution. In this context, Sandberg et al, (2002) proposed a method that utilizes Gabor filter-bank responses to generate vector-valued images, successively segmented by the ACWE model for vector-valued images (Chan et al, 2002). Paragios and Deriche, (1999) proposed a supervised texture segmentation method, in which a Gabor filter-bank is applied to the input and to a preferable pattern image. The filterbank responses are represented as multi-component conditional probability density functions and a textural feature vector encoding boundary information is generated. The maximum vector component is used to guide the GAC model. He et al, (2004) proposed an unsupervised texture segmentation method employing Geodesic Active Regions (Paragios, 2000) guided by the responses of a Gabor filter bank. Sagiv et al, (2004) proposed a method according to which the input image is filtered by a Gabor filter-bank to create a feature space and extract a two-dimensional 
Riemmannian manifold of local textural features via the Beltrami framework (Sochen et al, 1998). The textural feature vector generated by this method is used to guide the GAC model as well as an Integrated Active Contour (IAC) model extending both ACWE and GAC models.

The Gabor-based texture segmentation methods are credited as being state-of-the-art in texture analysis. However, despite their theoretical elegance and their psychovisual interpretation (Sagiv et al, 2004), these methods tend to be computationally demanding. Furthermore, Gabor filter responses are monotonic functions of gray-level intensities. This renders Gabor-based methods sensitive to changes of the illumination conditions (Mäenpää, 2003).

Liapis et al, (2004) and Aujol et al, (2003) introduced two supervised texture segmentation methods employing the Discrete Wavelet Frames Transform. Textural feature vectors extracted from the wavelet domain are utilized to guide level set active contours. Wavelet features are suitable for the representation of non-stationary textures. However, there are cases for which Gabor features result in better texture representation than wavelet features (Pichler et al, 1996; Aujol and Chan, 2006).

2) Statistical methods. Lehmann et al, (2001) proposed a supervised texture segmentation method that employs an active contour model guided by co-occurrence matrices. This method involves the application of the active contour model to prototype images, from which co-occurrence matrix features of target textures are extracted. Co-occurrence matrix features are also extracted from test images, and the similarity of the prototype and the testing image features is evaluated. The metrics, used to evaluate the similarity, weight linear combinations of the active contour model parameters used for the segmentation of the prototype images. The active contour model parameters resulting from these linear combinations are used for the segmentation of the test images. Pujol and Radeva, (2004) proposed a supervised method for learning the local appearance of the texture classes based on a set of co-occurrence matrix features. This method employs Fisher Linear Discriminant Analysis (Duda et al, 2001) to obtain an optimal reduced feature space. It applies a Gaussian mixture model to construct a likelihood map in which each pixel is been assigned the likelihood of representing each texture class. As a last step, they use a regularized version of the likelihood map to guide a generalized Gradient Vector Flow active contour model (Xu and Prince, 1998). The overhead introduced in the computation of co-occurrence matrix features as well as their moderate texture classification performance make them unappealing for incorporation to an active contour texture segmentation framework (Randen and Husoy, 1999).

Another statistical method for texture segmentation with active contour models has been proposed by Rousson et al, (2003). According to this method a seven-dimensional feature vector comprised of four gray-level intensity features and three features that capture texture orientation, is built. The gray-level intensity and the textural features are assumed to follow Parzen and Gaussian distributions, respectively. The distribution functions are embedded in a level set active contour model inspired from (Paragios and Deriche, 1999). Huang et al, (2004) introduced a new class of active contour models, metamorphs, which are formulated so as to integrate both shape and textural information. Metamorphs constitute a generalization of previous parametric and level set active contour models. They capture 
texture information using a nonparametric kernel-based approximation of the intensity probability density function inside the contour. Allili et al, (2004) modeled the intensity distribution of each image region by a mixture of Gaussian distributions and adopted the energy minimization approach introduced in (Kimmel, 2003). Lee et al, (2005) proposed a framework allowing the use of image feature statistics describing intensity distribution, orientation, polarity, anisotropy etc and applied a level set active contour model inspired by GAC and ACWE. The contour evolution follows an equation involving an edge stopping function, which is calculated as the inverse of the determinant of a metric tensor based on the Kullback-Leibler divergence. Awate et al, (2006) recently introduced a general active contour framework, which facilitates the exploitation of higher-order statistics derived from various textural features.

The Local Binary Pattern (LBP) operator, introduced by Ojala et al, (1996), is defined so as to provide a condensed encoding of local microstructures that captures textural information. It has been supported by various comparative studies on texture analysis (Ojala et al, 1996; Paclic et al, 2002; Mäenpää and Pietikäinen, 2004) which demonstrate that LBP texture representation can be superior to Gabor, wavelet and co-occurrence approaches, with a smaller computational overhead. Unlike the Gabor approach, which utilizes textural features calculated from the weighted mean of pixel values over a small neighborhood, the LBP operator considers each pixel in the neighborhood separately, providing even more fine-grained information. The textural features estimated using the LBP operator are invariant to any monotonic change in gray-level intensities, resulting in a robust representation of textures under varying illumination conditions and can be made multiscale and invariant against rotation (Mäenpää, 2003).

Recent applications utilizing the LBP distributions for texture representation include object detection (Zhang et al, 2006), realtime facial expression recognition (Feng et al, 2005), and landform segmentation of light detection and ranging imagery (Lucieer and Stein, 2005). Unsupervised texture segmentation algorithms utilizing the LBP distributions have been mainly based on hierarchical splitting and agglomerative merging (Ojala and Pietikäinen, 1999), as well as on region-competition approaches (Qing et al, 2005). However, such methods involve iterative calculations of histograms with potentially large numbers of bins that are computationally intensive and memory consuming (Ojala and Pietikäinen, 1999). This fact partially reverses the advantage of small computational overhead associated with the calculation of the LBP values. It should be noted that a texture segmentation method encompassing the advantages of both LBP texture representation methodology and of active contours, has not yet been proposed.

This study investigates LBP-guided active contour approaches to texture segmentation. In this context, two novel approaches have been formulated:

1. Scalar-LBP Active Contour (s-LAC)

2. Vector-LBP Active Contour ( $v$-LAC)

$s$-LAC encodes the spatial distribution of the most discriminative LBPs of an input image into gray-level intensities producing a new image which is subsequently segmented by ACWE model. This approach avoids the iterative 
calculation of active contour equation terms derived from textural feature vectors, and enables efficient, high quality texture segmentation. $v$-LAC utilizes multidimensional feature vectors representing LBP distributions that encode the textural properties of image regions rather than the single pixel information utilized in the case of vector-valued ACWE. Moreover, unlike vector-valued ACWE, the similarity between the LBP distributions is quantified by means of the log-likelihood statistic, which favors segmentations corresponding to image regions of minimum total entropy. Although computationally demanding, $v$-LAC can lead to higher segmentation quality than $s$-LAC. In order to reduce the computational cost involved in $v$-LAC segmentation the successive application of both $s$-LAC and $v$-LAC is proposed.

The rest of this paper is organized in five sections. Sections 2 and 3 outline the ACWE model and the LBP features, respectively. Section 4 describes the proposed s-LAC and $v$-LAC approaches for texture segmentation. The results from the experimental evaluation of the proposed approaches on textures and natural scenes acquired from standard image databases are apposed in Section 5. Finally, in Section 6 the conclusions of this study are summarized.

\section{Active Contour Without Edges}

The vector-valued ACWE model as posed in (Chan et al, 2002), follows the Mumford-Shah segmentation approach (Mumford and Shah, 1989) and has the form of a minimization problem: if we consider $\Omega$ as a bounded open subset of $R^{2}$, with $\partial \Omega$ the boundary, we seek for the infimum of the energy functional $F$ :

$$
F\left(C, \bar{c}_{+}, \bar{c}_{-}\right)=\mu \cdot \text { length }(C)+\int_{\text {inside(C) }} \frac{1}{b} \sum_{i=1}^{b} \lambda_{i}^{+}\left|u_{0}^{i}(x, y)-c_{+}^{i}\right|^{2} d x d y+\int_{\text {outside(C) }} \frac{1}{b} \sum_{i=1}^{b} \lambda_{i}^{-}\left|u_{0}^{i}(x, y)-c_{-}^{i}\right|^{2} d x d y
$$

where $u_{0}^{i}, i=1, \ldots, b$ are the components that describe the original image $u_{0}, C(s):[0,1] \rightarrow R^{2}$ is a parameterized curve, $c_{+}^{i}$ and $c_{-}^{i}, i=1,2, \ldots, b$ are unknown constants representing the average value of $u_{0}^{i}$ inside and outside the curve, and parameters $\mu, \lambda_{i}^{+}$and $\lambda_{i}^{-}, i=1, \ldots, b$ are weights for the regularizing term and the fitting terms, respectively. Each component $u_{0}^{i}(x, y), i=1, \ldots, b$, is defined over a single pixel $(x, y)$. For example in (Chan et al, 2002), each $u_{0}^{i}(x, y), i=$ 1, 2, 3 represent a component of the RGB color space at pixel $(x, y)$. The foreground and the background regions resulting from the segmentation of the image by the contour $C$, are denoted as "inside $C$ " and "outside $C$ ", respectively.

The vector-valued ACWE model uses the level set method (Osher and Sethian, 1988) which provides an efficient means for moving curves and surfaces, on a fixed regular grid, allowing for automatic topology changes, such as merging and splitting. Following (Osher and Sethian, 1988), the curve $C$ is represented implicitly, by the zero level set of a Lipschitz function $\phi: \Omega \rightarrow R$, such that: 


$$
\begin{aligned}
& C=\{(x, y) \in \Omega: \phi(x, y)=0\}, \\
& \text { inside }(C)=\{(x, y) \in \Omega: \phi(x, y)>0\}, \\
& \text { outside }(C)=\{(x, y) \in \phi(x, y)<0\}
\end{aligned}
$$

Using the one-dimensional Dirac measure $\delta$ and the Heaviside function $H$, defined by:

$$
\delta(z)=\frac{d}{d z} H(z), H(z)= \begin{cases}1, & \text { if } z \geq 0 \\ 0, & \text { if } z<0\end{cases}
$$

the level set formulation of the energy functional $F$ is:

$$
F\left(\bar{c}_{+}, \bar{c}_{-}, \phi\right)=\mu \cdot \int_{\Omega} \delta(\phi(x, y))|\nabla \phi(x, y)| d x d y+\int_{\Omega} \frac{1}{b} \sum_{i=1}^{b} \lambda_{i}^{+}\left|u_{0}^{i}(x, y)-c_{+}^{i}\right|^{2} H\left(\phi(x, y) d x d y+\int_{\Omega} \frac{1}{b} \sum_{i=1}^{b} \lambda_{i}^{-}\left|u_{0}^{i}(x, y)-c_{-}^{i}\right|^{2}(1-H(\phi(x, y))) d x d y\right.
$$

By minimizing $F\left(\bar{c}_{+}, \bar{c}_{-}, \phi\right)$ with respect to the unknown constant vectors $c_{+}^{i}$ and $c_{-}^{i}$, the following relations are obtained:

$$
\begin{gathered}
c_{+}^{i}(\phi)=\frac{\int_{\Omega} u_{0}^{i}(x, y) H(\phi(x, y)) d x d y}{\int_{\Omega} H(\phi(x, y)) d x d y} \\
c_{-}^{i}(\phi)=\frac{\int_{\Omega} u_{0}^{i}(x, y)(1-H(\phi(x, y))) d x d y}{\int_{\Omega} H(\phi(x, y)) d x d y}
\end{gathered}
$$

which represent the averages of $u_{0}^{i}$ inside and outside the curve $C$ respectively.

By keeping $c_{+}^{i}$ and $c_{-}^{i}$ fixed and by minimizing $F$ with respect to $\phi$, we deduce the associated Euler-Langrange equation for $\phi$. Parameterizing the descent direction by an artificial time $t \geq 0$, the equation in $\phi(t, x, y)$ (with $\phi(0, x, y)=\phi_{0}(x, y)$ defining the initial contour $)$ is:

$$
\frac{\partial \phi}{\partial t}=\delta(\phi)\left[\mu \cdot \operatorname{div}\left(\frac{\nabla \phi}{|\nabla \phi|}\right)-\frac{1}{b} \cdot \sum_{i=1}^{b} \lambda_{i}^{+}\left(u_{0}^{i}-c_{i}^{+}\right)^{2}+\frac{1}{b} \cdot \sum_{i=1}^{b} \lambda_{i}^{-}\left(u_{0}^{i}-c_{i}^{-}\right)^{2}\right]=0
$$

where a smooth approximation of the Heaviside function $H$ is used, as in (Chan et al, 2002). Starting with an initial contour, defined by $\phi_{0}$, at each time step the vector averages $c_{+}^{i}$ and $c_{-}^{i}$ are updated and $\phi$ evolves according to the partial differential equation (7). Equations (1)-(7) describe the original, scalar ACWE model (Chan and Vese, 2001) for $b=1$. More details for the numerical aspects of the level set evolution can be found in (Aubert and Vese, 1997).

\section{Local Binary Patterns}

The LBP operator, as defined in (Ojala et al, 2002), utilizes a binary representation of local texture patterns. Let $T$ be such a texture pattern, defined in a local neighborhood of a gray-level texture image as the joint distribution of the gray-levels of $P(P>1)$ image pixels:

$$
T=\tau\left(g, g_{0}, \ldots, g_{P-1}\right)
$$


where $g$ is the gray-level of the central pixel of the local neighborhood and $g_{p}(p=0, \ldots, P-1)$ represents the gray-level of $P$ equally spaced pixels arranged on a circle of radius $R(R>0)$, forming a circularly symmetric neighbor set. Assuming that the differences $g_{p}-g$ are not affected by changes in mean luminance $g$, the joint difference distribution $\tau\left(g_{0}-g, \ldots, g_{P-1}-g\right)$ is invariant against gray-level shifts. Moreover, the LBP approach achieves invariance with respect to the scaling of the gray-levels by considering $H\left(g_{p}-g\right)$ instead of $g_{p}-g$, i.e. the joint signed difference distribution $T$ :

$$
T^{\prime}=\tau\left(H\left(g_{0}-g\right), \ldots, H\left(g_{P-1}-g\right)\right)
$$

The LBP encoding is obtained by assigning a binomial factor $2^{p}$ to each term $H\left(g_{p}-g\right)$. A unique $L B P_{P, R}$ value that encodes the spatial structure of the local image texture $T^{\prime}$ is estimated by:

$$
L B P_{P, R}=\sum_{p=0}^{P-1} H\left(g_{p}-g\right) 2^{p}
$$

The distribution of the $L B P_{P, R}$ values calculated over an image region, comprises a highly discriminative feature vector for texture segmentation, as demonstrated in various comparative studies (Ojala et al, 1996; Paclic et al, 2002; Mäenpää and Pietikäinen, 2004). More detailed information concerning the LBP can be found in (Mäenpää and Pietikäinen, 2004).

\section{LBP-Guided Active Contours}

\subsection{Scalar-LBP Active Contour (s-LAC)}

The underlying idea of $s$-LAC is to encode the spatial distribution of the most discriminative $L B P_{P, R}$ values of an input image into gray-level intensities so as to produce a new image that satisfies the assumption of approximately piecewise constant intensities. This is the basic assumption of the ACWE model, which is subsequently applied on the new image. As this approach avoids the iterative calculation of active contour equation terms derived from textural feature vectors, $s$-LAC can be more efficient than other active contour approaches.

$s$-LAC algorithm begins with the calculation of the $L B P_{P, R}$ values of all pixels of the input image $I$. A binary image is assigned to each of the existent LBP values. For each $i=L B P_{P, R}(x, y)$, the pixel $(x, y)$ of the binary image $B_{i}$ is labeled white, indicating the presence of the LBP value $i$, otherwise it is labeled black.

In the sequel, each $B_{i}$ is divided into constant-sized blocks and the occurrence probability $P_{\text {pvalue }}(i, j)$ of the pixel value pvalue (white or black) in each block $j$ of $B_{i}$ is estimated. Since white pixels in $B_{i}$ indicate the presence of the LBP value $i$, their density may vary for regions of different texture, characterized by different LBP distributions. The conditional entropy $H_{i}$, given an LBP value $i$ :

$$
H_{i}=-\sum_{\text {pvalue }} \sum_{j} P_{\text {pvalue }}(i, j) \cdot \log _{2} P_{\text {pvalue }}(i, j)
$$

can be used to evaluate the texture discrimination capability of the LBP value $i$. From an information-theoretic point of 
view, eq. (11) is highly intuitive (Hermes and Buhmann, 2003). Since the conditional entropy $H_{i}$ of an image with respect to an LBP value $i$, measures the level of uncertainty about this value, $H_{i}$ is expected to be smaller if white pixels are mainly concentrated in some image blocks. Thus, a small value for conditional entropy $H_{i}$ indicates that the corresponding LBP value $i$ is dominant on an image region.

The binary images $B_{i}, i=1,2, \ldots 2^{P}$ are sorted according to their conditional entropy $H_{i}$, and the $r$ top-ranked images are selected. The logical OR operator is applied on the $K=2^{r}-1$ non-empty combinations of the selected $r$ binary images. The resulting "cumulative" binary images $C B_{k}, k=1,2, \ldots K$, contain information derived from subsets of the existent LBPs. This is in agreement with (Mäenpää, 2003), according to which an appropriately selected subset of LBPs maintains most of the textural information associated with the set of the existent LBPs. The "cumulative" binary image $C B_{M}$ with the minimum conditional entropy is selected, according to:

$$
M=\underset{k}{\arg }\left(\min \left(H_{k}\right)\right)
$$

Equation (12) imposes that the selected "cumulative" image $\mathrm{CB}_{M}$ will be comprised of regions characterized by distinguishable white pixel densities.

In order to limit the effect of local variances in the spatial frequency of the LBPs, a Gaussian kernel $W_{G}$ is convolved with $C B_{M}$. This results in a smoothed image $C B_{G}$ of nearly homogeneous image regions, which satisfy the assumption of ACWE model for piecewise constant intensities. Such smoothing operations have been proved to enhance texture discrimination, as the notion of texture is undefined at the single pixel level and is always associated with some set of pixels (Unser and Eden, 1990). The convolution with the Gaussian kernel $W_{G}$ ensures that the gray level of each pixel in the smoothed image $C B_{G}$ depends on the distances of the LBPs, which are present in the neighborhood of the pixel and have been associated with the texture of interest in the previous steps of the algorithm. Furthermore, smoothing accelerates the convergence of the subsequently applied active contour (Akgul and Kambhamettu, 2003).

In the last step of the algorithm, the ACWE model is applied to $C B_{G}$. The region-based formulation of this active contour model enables the segmentation of an image into two discrete regions, even if these regions are not explicitly defined by high intensity gradients. In addition, the level set formulation of the ACWE model allows its adaptation to topological changes, such as splitting or merging, in case regions of the same texture are interspersed in the image.

The steps of the proposed algorithm can be summarized as follows:

1. Calculate $L B P$ values

For each pixel $(x, y)$ in I Calculate $L B P_{P, R}(x, y)$

2. Generate binary images $B_{i}, i=1,2, \ldots, 2^{P}$

Initialize $B_{i}(x, y)=0$

For each $L B P_{P, R}(x, y)$ do

$$
i=L B P_{P, R}(x, y)
$$




$$
B_{i}(x, y)=1
$$

End

3. Generate "cumulative" binary image $C B_{M}$

Rank all $B_{i}$ according to $\xi_{i}$

For each combination $C O M B_{k}=\left\{B_{i 1}, B_{i 2}, \ldots, B_{i l}\right\}, k=1,2, \ldots K, I=\operatorname{card}\left(C O M B_{k}\right)$ of the $r$ top-ranked $B_{i}$ do

$$
C B_{k}=\left(B_{i 1} \text { OR } B_{i 2} \text { OR } \ldots \text { OR } B_{i l}\right)
$$

End

Find $C B_{M}$ using (12)

4. Smoothing and Segmentation

$C B_{G}=C B_{M}^{*} W_{G}$

Segment $C B_{G}$ using ACWE.

Figure 1 illustrates a schematic representation of the algorithm step by step, as applied on a composite image of the Brodatz collection (Brodatz, 1996). Figure 2 illustrates the set $C O M B_{i}$ of the binary images $B_{i 1}, B_{i 2}, B_{i 3}, B_{i 4}$ (generated at step 2) that were used to generate the cumulative image $C B_{M}$ at the third step of the algorithm. 

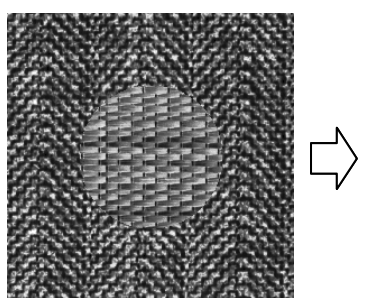

Input image

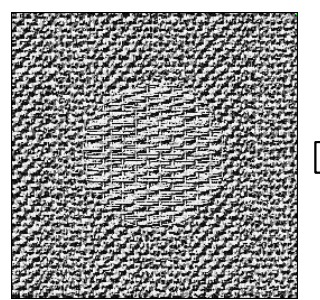

$\operatorname{LBP}_{P, R}(x, y) \forall(x, y)$ Step 1

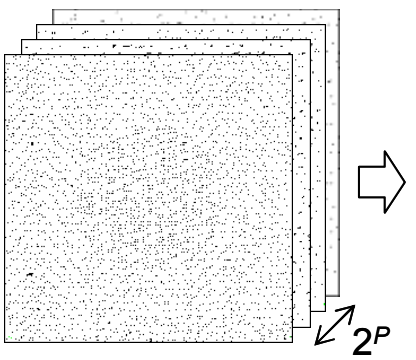

Binary images $B_{i}$

Step 2

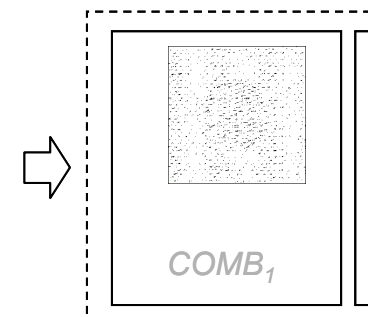

$C B_{1}$ (1) $\mathrm{CB}_{2}$

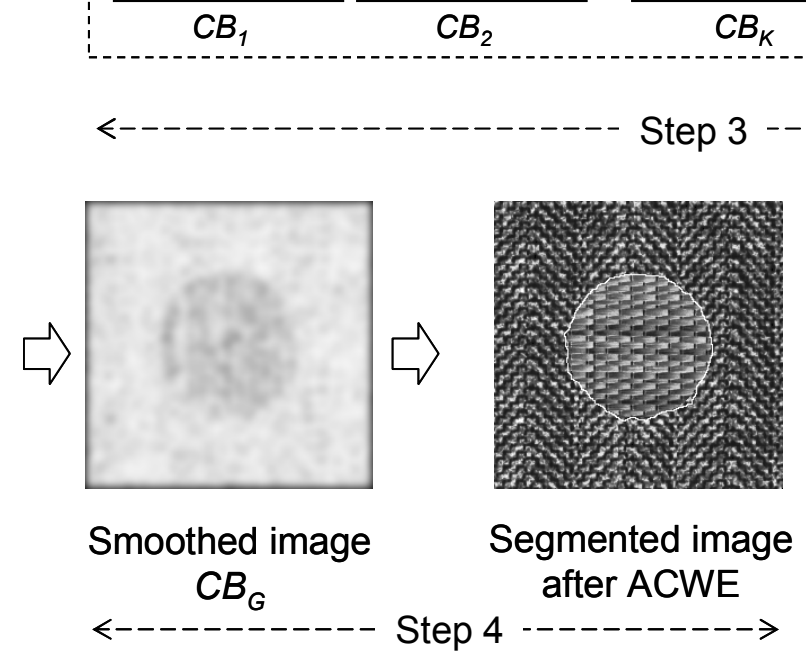

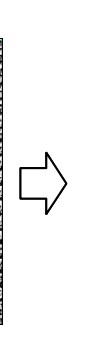

(1)

Fig. 1. Schematic representation of the steps of the $s$-LAC algorithm and images generated at each step. The input image is Brodatz D17D55. The binary, the cumulative, and the smoothed images have been inverted for illustrational purposes.
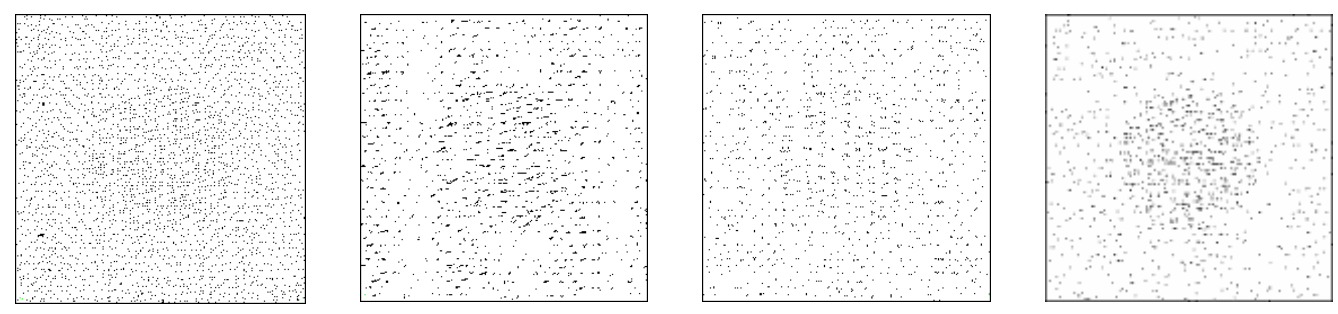

Fig. 2. Four binary images $B_{i 1}, B_{i 2}, B_{i 3}, B_{i 4}$ that were used to generate the cumulative image $C B_{M}$ illustrated in Fig. 1 . The images have been inverted for illustrational purposes.

\subsection{Vector-LBP Active Contour (v-LAC)}

In this section, we introduce an alternative LBP-guided active contour approach, $v$-LAC. As in the case of $s$-LAC, $v$ LAC is formulated acknowledging that texture is undefined at the single pixel level and it is always associated with an 
image region (Unser and Eden, 1996). It utilizes vectors $D^{i}(x, y), i=1,2, \ldots, b$, where each component $D^{i}(x, y)$ corresponds to the $i$-th bin of the LBP distribution, and $b=2^{P}$ is the number of bins comprising each distribution. $D^{i}(x$, $y$ ) encodes the textural properties of $k \times k$-pixel image regions centered at pixel $(x, y)$.

In order to incorporate the regional information encoded by means of LBP distributions into (1), we consider the replacement of the vector $u_{0}(x, y)$ which represents the image components at a single pixel, with $D^{i}(x, y)$. Moreover, motivated by Ojala et al, (2002) in which the log-likelihood statistic is suggested as an accurate similarity measure for LBP distributions, we consider the replacement of $\left|u_{0}^{i}(x, y)-c_{+}^{i}\right|^{2}$ and $\left|u_{0}^{i}(x, y)-c_{-}^{i}\right|^{2}$ in (1), with $\left(1-D^{i}(x, y) \log \left(c_{+}^{i}\right)\right)$ and $\left(1-D^{i}(x, y) \log \left(c_{-}^{i}\right)\right)$ respectively. These considerations lead to the derivation of a new energy functional:

$$
F^{\prime}\left(C, \bar{c}_{+}, \bar{c}_{-}\right)=\mu \cdot \operatorname{length}(C)+\int_{\text {inside }(C)} \frac{1}{b} \sum_{i=1}^{b} \lambda_{i}^{+}\left(1-D^{i}(x, y) \log \left(c_{+}^{i}\right)\right) d x d y+\int_{\text {outside }(C)} \frac{1}{b} \sum_{i=1}^{b} \lambda_{i}^{-}\left(1-D^{i}(x, y) \log \left(c_{-}^{i}\right)\right) d x d y
$$

The Euler-Langrange formulation of (13) is:

$$
\frac{\partial \phi}{\partial t}=\delta(\phi)\left[\mu \cdot \operatorname{div}\left(\frac{\nabla \phi}{|\nabla \phi|}\right)-\frac{1}{b} \cdot \sum_{i=1}^{b} \lambda_{i}^{+}\left(1-D^{i}(x, y) \log \left(c_{+}^{i}\right)\right)+\frac{1}{b} \cdot \sum_{i=1}^{b} \lambda_{i}^{-}\left(1-D^{i}(x, y) \log \left(c_{-}^{i}\right)\right)\right]=0
$$

where $\phi$ is the level set function, implicitly representing curve $C$.

\section{Experimental Results}

Experiments were performed to investigate the performance of the proposed LBP-guided active contours on texture segmentation. The dataset used is comprised of composite texture images from the Brodatz album (Brodatz, 1996), and natural scenes from the Vistex (MIT Media Lab), Li and Wang (Li and Wang, 2003) and the Berkeley (Martin et al, 2001) databases (examples illustrated in Fig. 3). The size of each image of the dataset used was $256 \times 256$. The active contour algorithms were implemented in Microsoft Visual $\mathrm{C}++$ and executed on a $3.2 \mathrm{GHz}$ Intel Pentium IV workstation. Densely distributed small circular contours were used for the initialization of the algorithms. As a segmentation quality measure we have considered the overlap $q$ :

$$
q=\frac{A \cap G}{A \cup G}
$$

where $A$ is the region delineated by the algorithm and $G$ is the ground truth region. However, this measure has been estimated only for the segmentations of composite texture images, for which the ground truth regions are explicitly defined.

The results are organized in three parts. The first two parts present the results obtained by $s$-LAC and $v$-LAC approaches, whereas the third part compares the performance of the proposed LBP-guided active contours with the performance achieved by a baseline segmentation algorithm and state of the art active contours reported in the literature. 


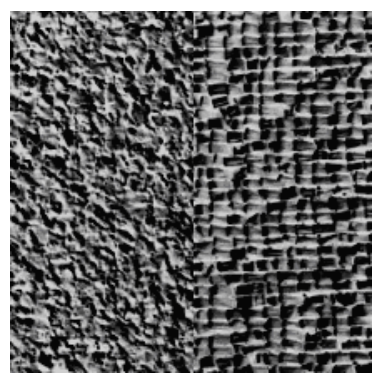

(a)

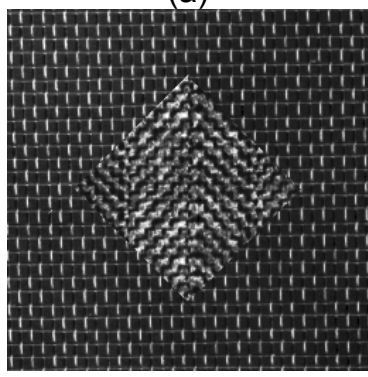

(e)

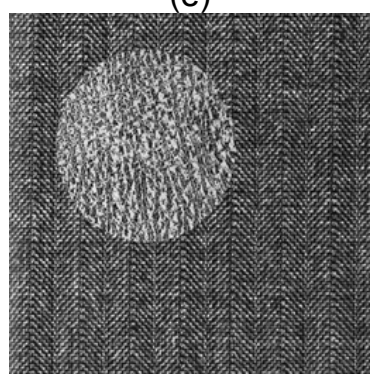

(i)

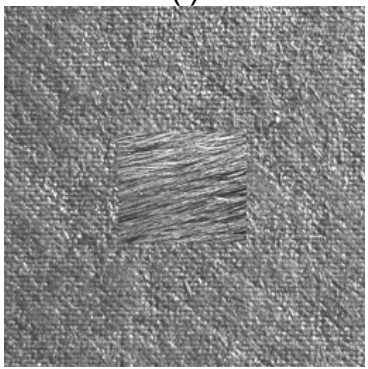

(m)

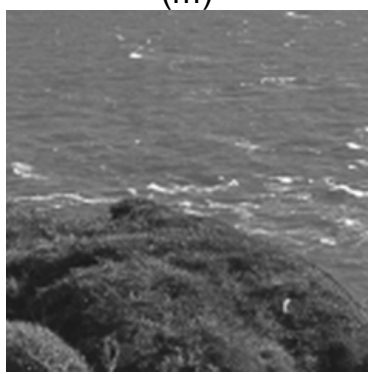

(q)

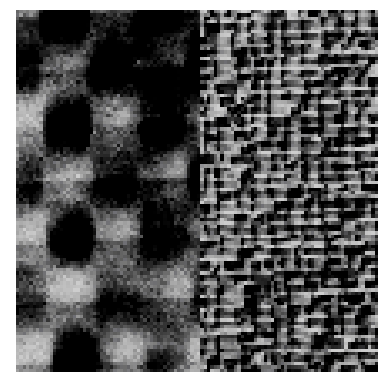

(b)

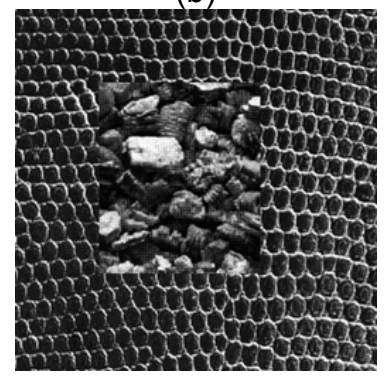

(f)

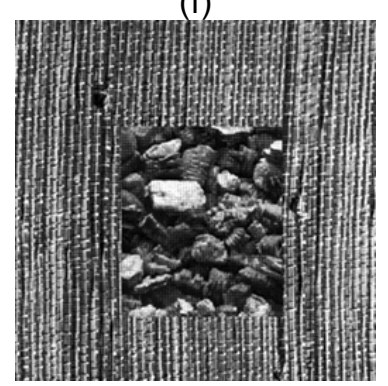

(j)

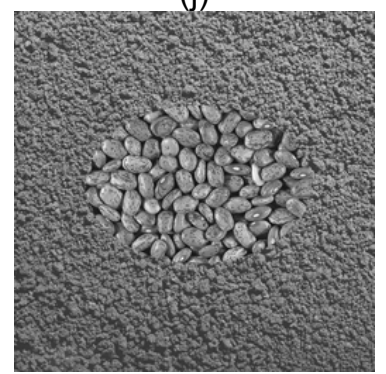

(n)

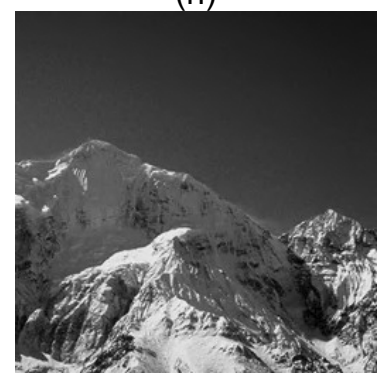

(r)

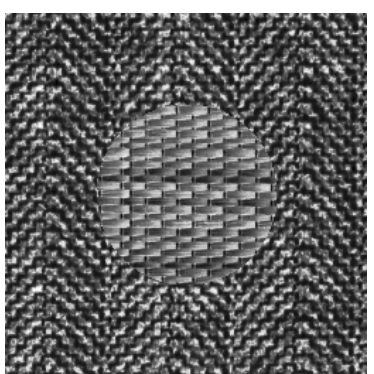

(c)

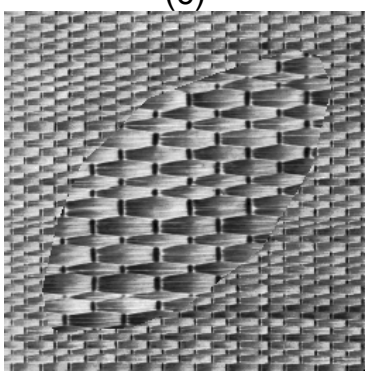

(g)

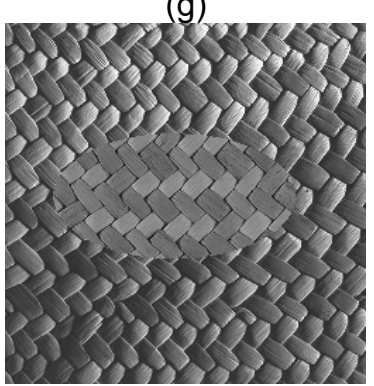

(k)

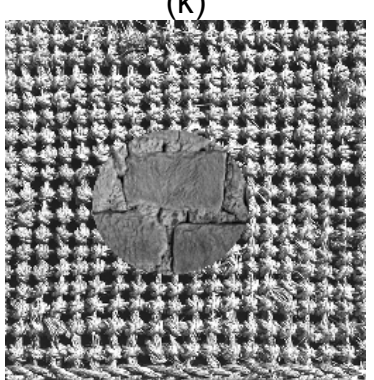

(o)

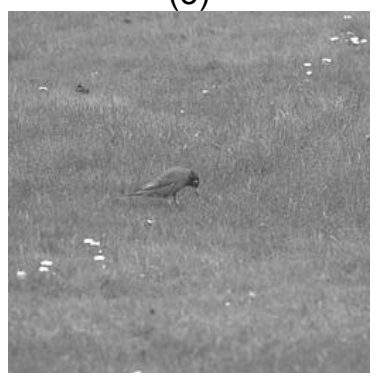

(s)

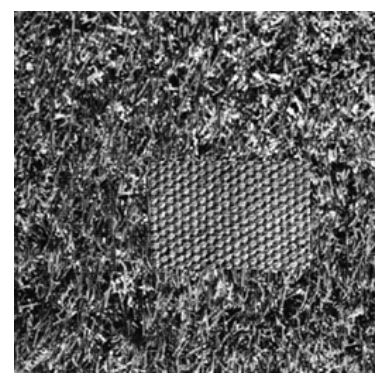

(d)

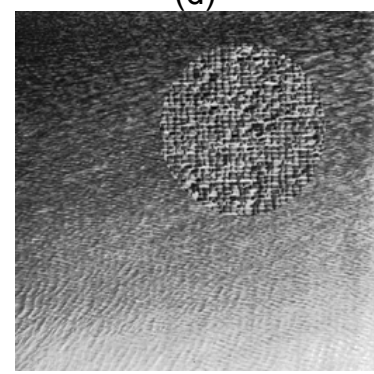

(h)

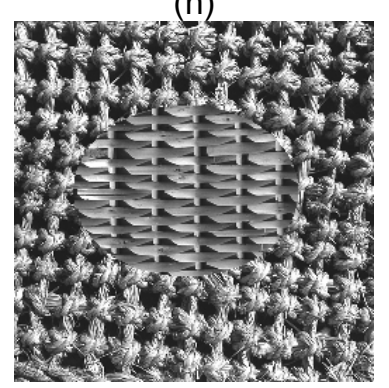

(I)

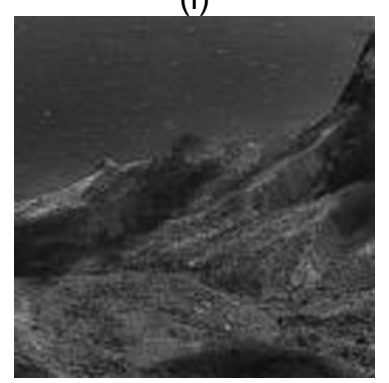

(p)

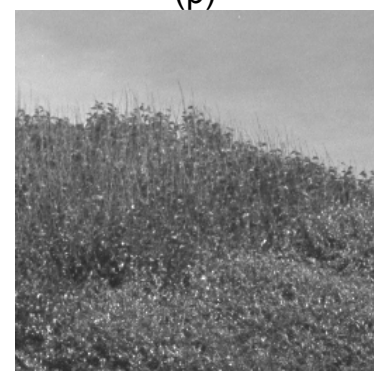

(t)

Fig. 3. Test images from the dataset used in the experiments: (a) Brodatz D4D84, (b) Brodatz D8D84 (c) Brodatz D17D55, (d) Brodatz D9D77, (e) Brodatz D6D17, (f) Brodatz D3D5, (g) Brodatz D56D56 (re-scaled), (h) Brodatz D38D106, (i) Brodatz D17D24, (j) Brodatz D79D5, (k) Vistex Fabric2Fabric1, (l) Vistex Fabric10Fabric13, (m) Vistex Fabric5Fabric7, (n) Vistex Food0Food5 (o) Vistex Fabric09Brick05 (p) Vistex ValleyWater1, (q) Vistex GroundWaterCity1, (r) Wang 804, (s) Berkeley 28096, and (t) Vistex GrassPlantSky6. 
$5.1 \quad$ s-LAC segmentation results

The LBP operator considered in the experiments with $s$-LAC was $L B P_{8,1}$. The parameters involved were determined by performing preliminary segmentation experiments on a randomly selected subset of the available dataset (comprised of the images (a)-(d) of Fig. 3 ). The block size was set at $16 \times 16$ pixels, as this provided the highest average overlap on this subset. Moreover, a total of $r=5$ top-ranked binary images in the third step of the $s$-LAC algorithm was found to be sufficient for the performed segmentation tasks, as for $r>5$ a marginal improvement of the segmentation quality was observed.

A rough grid search of the parameter space of the active contour was performed on the subset of images selected for parameter tuning. Subsequent grid searches were performed for fine tuning within smaller ranges around the highest average parameter values obtained from the rough parameter search. The highest average overlap was obtained for $\lambda^{+}=\lambda^{-}=5$ and $\mu=0.01 \cdot 256^{2}$. The values of the parameters $\lambda^{+}$and $\lambda^{-}$were considered to be equal to each other, and the values of $\mu$ where considered proportional to the image size, in accordance with (Chan and Vese, 2001). It was observed that a variation of approximately $10 \%$ of $\lambda^{+}$and $\lambda^{-}$, and of approximately $30 \%$ of $\mu$ around the values used results in overlap values that differ no more than $1.0 \%$. This indicates that slight perturbations of $\lambda^{+}$ and $\lambda^{-}$have a greater impact on the obtained overlaps than slight perturbations of $\mu$.

Figure 4 illustrates example segmentation results obtained by the application of $s$-LAC on the images of Fig.3. The overlaps measured are presented in Table 1. Their average overlap is estimated to be $95.2 \pm 2.9 \%$. The results show that s-LAC managed to segment all the composite Brodatz images accurately. It is worth noting that the segmentation is quite satisfying even for the Brodatz images D3D5, D38D106 and D79D5 illustrated in Fig. 4f, 4h and 4h respectively, which contain non-stationary textures.

Table 1

Overlaps obtained by the s-LAC algorithm for the segmentation of the images illustrated in Fig. 3 . .

\begin{tabular}{cccccc}
\hline Image & Overlap (\%) & Image & Overlap (\%) & Image & Overlap (\%) \\
& & & & & \\
\hline a & 99.0 & $\mathrm{f}$ & 87.5 & $\mathrm{k}$ & 96.7 \\
\hline $\mathrm{b}$ & 99.2 & $\mathrm{~g}$ & 98.5 & $\mathrm{I}$ & 97.1 \\
\hline $\mathrm{c}$ & 96.1 & $\mathrm{~h}$ & 93.1 & $\mathrm{~m}$ & 94.3 \\
\hline $\mathrm{d}$ & 93.9 & $\mathrm{i}$ & 95.6 & $\mathrm{n}$ & 92.8 \\
\hline $\mathrm{e}$ & 95.4 & $\mathrm{j}$ & 94.7 & $\mathrm{O}$ & 94.2
\end{tabular}

The segmentations obtained by the application of s-LAC on the natural scenes illustrated in Figs. 40-4t, 
demonstrate the robustness of $s$-LAC to illumination changes, which is mainly attributed to the LBP texture representation. It can be observed that the textures of natural scenes are generally less uniform than the homogeneous textures of the test mosaics. In addition, due to the infinite scale of texture differences present in such images, choosing the right scale is a very subjective matter. For these reasons, there is often no 'correct' segmentation for a natural scene (Ojala and Pietikäinen, 1999). The bird illustrated in Fig. 4s has been successfully localized by $s-L A C$, although it occupies a rather small portion of the image. However, the roughly defined boundaries indicate that the statistical sample of LBPs within the bird's region may not be sufficient to differentiate its textural content from the surrounding background.

The convergence times of $s$-LAC observed for the available images, range between 3 and 4 seconds depending on the complexity of the target boundaries.

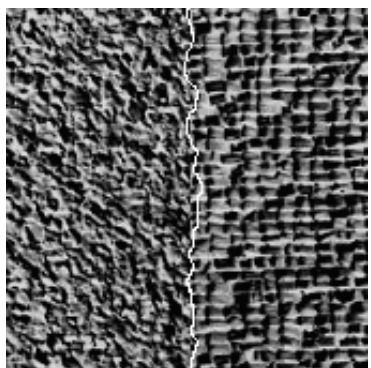

(a)

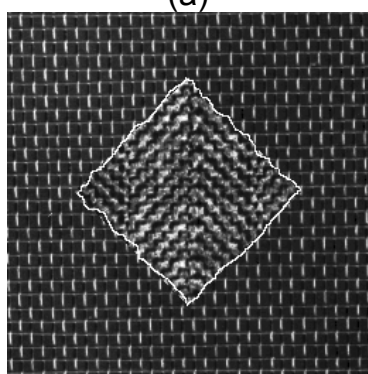

(e)

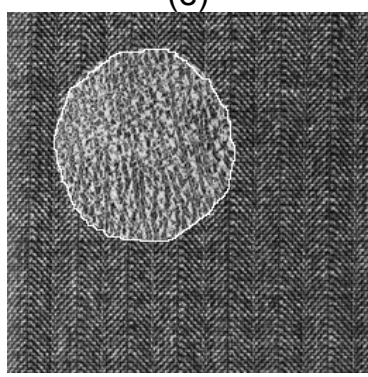

(i)

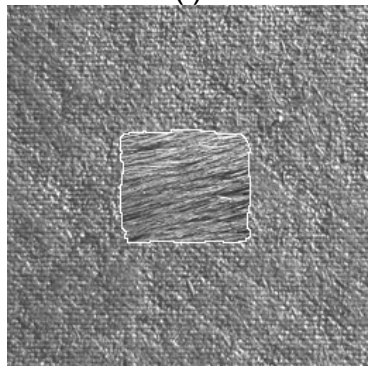

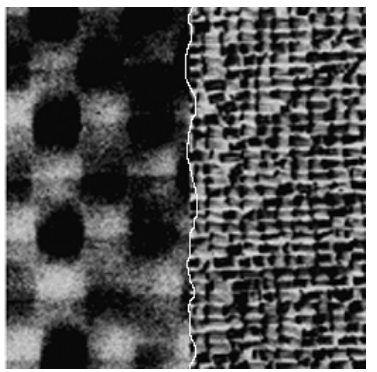

(b)

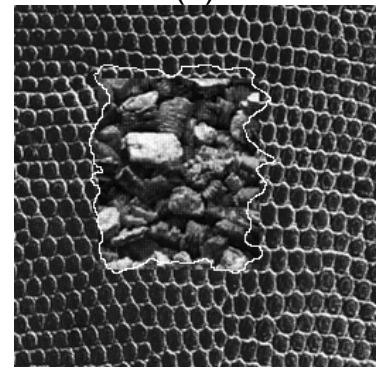

(f)

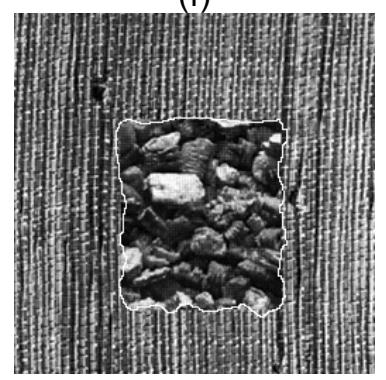

(j)

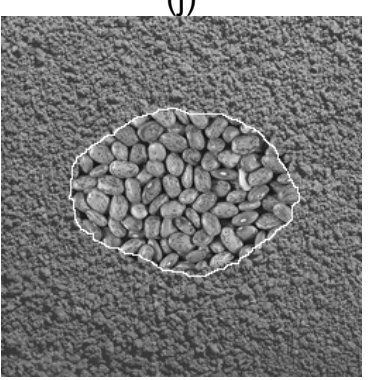

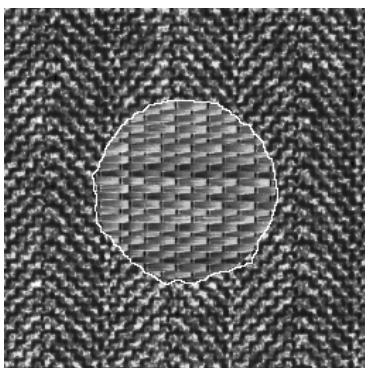

(c)

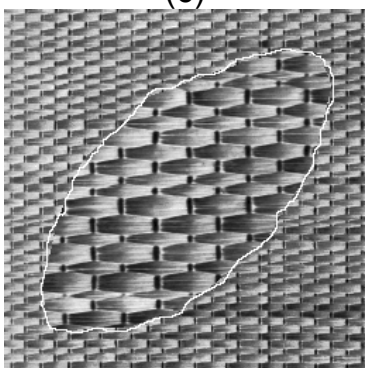

(g)

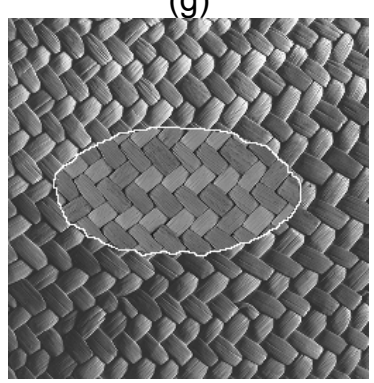

(k)

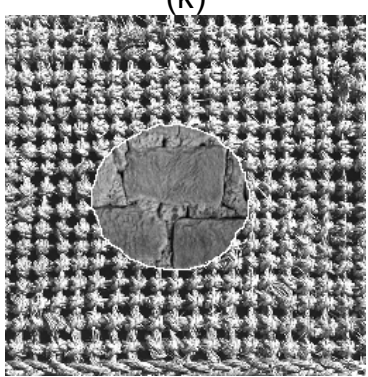

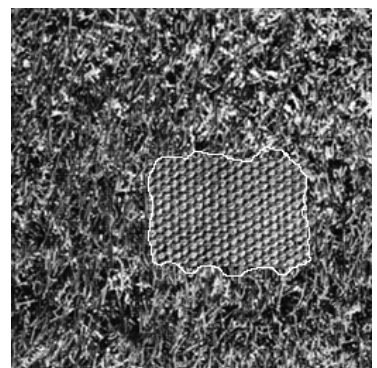

(d)

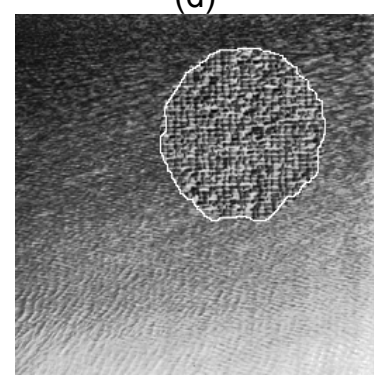

(h)

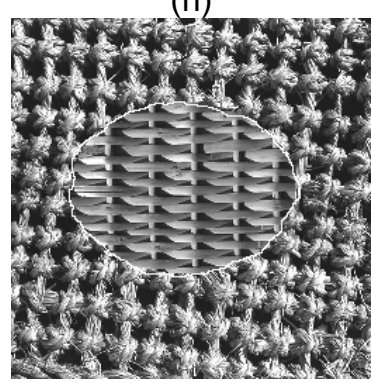

(I)

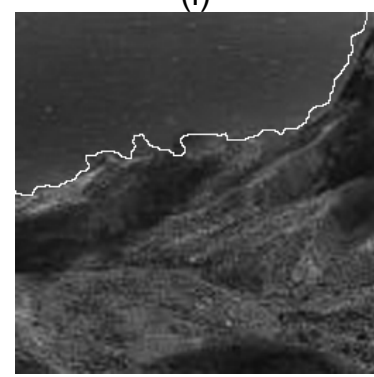


(m)

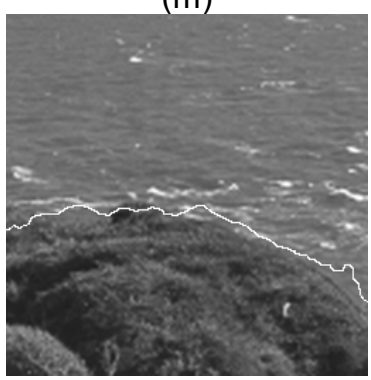

(q) (n)

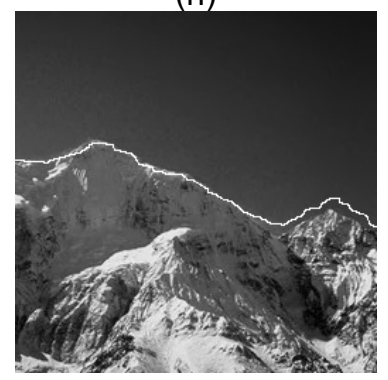

(r) (o)

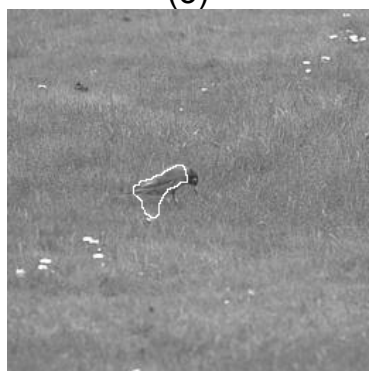

(s) (p)

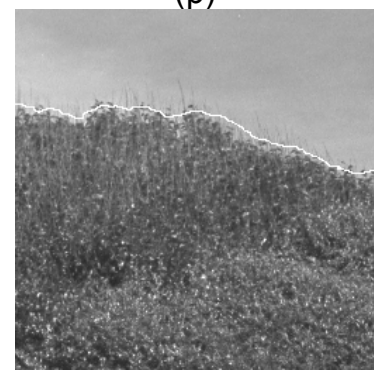

(t)

Fig. 4. Segmentation of the images illustrated in Fig. 3 using the s-LAC algorithm.

\section{$5.2 v$-LAC segmentation results}

By definition $v$-LAC utilizes feature vectors comprising LBP distributions. Equation (14) shows that the dimensionality of the LBP feature vectors affects negatively the performance of $v$-LAC. In the case of the $L B P_{8,1}$ operator the feature vectors are 256-dimensional. Such a high dimensionality can prove $v$-LAC almost impractical for the segmentation of typical images in terms of time performance. However, a considerable speedup of $v$-LAC, resulting in a marginal loss of precision, can be achieved by utilizing the $L B P_{4,1}$ instead of the $L B P_{8,1}$ operator. The $L B P_{4,1}$ results in 16-dimensional feature vectors, efficiently, without any overhead introduced by additional dimensionality reduction techniques (Mäenpää et al, 2003). A rule of thumb suggests that the number of entries for each bin of a histogram should be at least 10. Considering that the $L B P_{4,1}$ produces a 16-bin histogram, the number of entries required for the whole histogram is at least $16 \times 10=160$. Therefore, $k=13$ corresponds to the minimum $k \times k$ neighborhood that satisfies this requirement $\left(13^{2}=169>160\right)$.

The grid search approach of the parameter space described in Section 5.1 for the case of $s$-LAC led to the following set of parameter values: $\lambda_{+}^{i}=\lambda_{-}^{i}=7.5 \cdot 10^{5}, i=1,2, . . b$ and $\mu=0.1 \cdot 256^{2}$. It was observed that a variation of approximately $5 \%$ of $\lambda^{+}$and $\lambda^{-}$, and of approximately $20 \%$ of $\mu$ around the values used, results in overlap values that differ no more than $1.0 \%$. As in the case of $s$-LAC this indicates that slight perturbations of $\lambda^{+}$and $\lambda^{-}$have a greater impact on the obtained overlaps than slight perturbations of $\mu$. However, these estimates show that $v$-LAC is more sensitive to parameter perturbations than s-LAC.

The segmentation results obtained by the application of $v$-LAC on the available set of test images, using the $L B P_{4,1}$ operator, are illustrated in Fig. 5. The overlaps measured using v-LAC are presented in Table 2. These values are consistently higher than the overlaps obtained by the application of $s$-LAC on the same images, with an average value of $97.2 \pm 1.6 \%$. A remarkable improvement in the segmentation quality has been achieved by $v$-LAC in the case of Fig.

5f. This result indicates that $v$-LAC segmentation is less affected by non-stationarity of the textures characterizing different image regions. 
Overlaps obtained by the $v$-LAC algorithm for the segmentation of the images illustrated in Fig. 3.

\begin{tabular}{cccccc}
\hline Image & Overlap (\%) & Image & Overlap (\%) & Image & Overlap (\%) \\
& & & & & \\
\hline a & 99.8 & f & 96.4 & $\mathrm{k}$ & 97.9 \\
\hline b & 99.7 & g & 97.8 & l & 97.3 \\
\hline c & 97.5 & h & 96.7 & m & 96.4 \\
\hline d & 94.7 & i & 99.3 & n & 95.3 \\
\hline e & 96.2 & j & 97.3 & o & 95.3 \\
\hline
\end{tabular}

It is worth noting that the utilization of the log-likelihood distance in the formulation of $v$-LAC, instead of the Euclidean distance, resulted in a considerable enhancement of the segmentation quality which reached $10 \%$.

The segmentations obtained by the application of $v$-LAC on natural scenes are also of higher quality, when compared to the respective s-LAC segmentations. The boundary of the ground illustrated in Figs. $5 p-5 t$ have been delineated in a tighter fashion by $v$-LAC. Furthermore, unlike $s$-LAC in Fig. $4 \mathrm{~s}$, no leakage is observed in the bird's boundaries determined by $v$-LAC in Fig. $5 \mathrm{~s}$. The bird's head has been reasonably excluded from the segmented region, as it is evidently dissimilar to the rest of the bird's body.

Although $v$-LAC outperforms $s$-LAC in terms of segmentation quality, its computational requirements raise up to an order of magnitude for the particular experimental setup with convergence times ranging between 40 and 60 seconds.

$v$-LAC segmentation can be accelerated by successively applying both $s$-LAC and $v$-LAC. First, a fast, however accurate, segmentation can be obtained by the application of $s$-LAC. The level-set function resulting from s-LAC at convergence can be subsequently used for the initialization of $v$-LAC. $v$-LAC will then proceed to a fine-grained texture segmentation in a relatively small number of iterations. The overall segmentation times observed for the successive application of $s$-LAC and $v$-LAC are reduced up to 20 seconds, whereas the individual overlaps obtained for the segmentation of the composite texture images are approximately the same with the ones obtained with $v$-LAC with a divergence per image of $0.1 \%$ on average. The average overlap obtained is $97.2 \pm 1.5 \%$. This is a consequence of the fact that $v$-LAC is practically invariant to initialization and obtains almost identical segmentation results either by an initialization of densely distributed small circular contours or by an initialization derived from the segmentation result of $s$-LAC. This is achieved, as in the case of ACWE, by utilizing the regularized heaviside function $H$, suggested by Chan and Vese, (2001) to facilitate convergence to a global minimizer. 


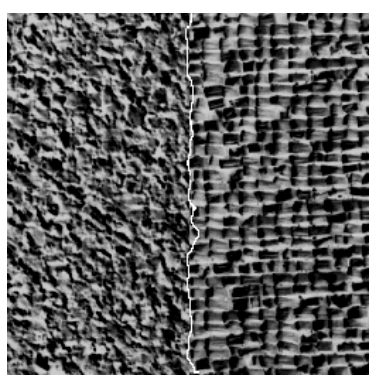

(a)

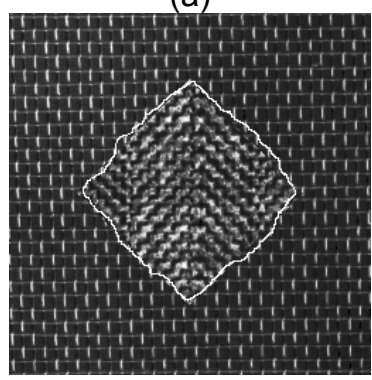

(e)

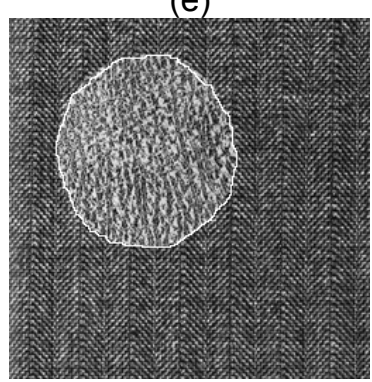

(i)

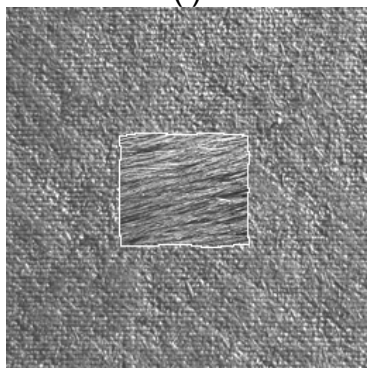

(m)

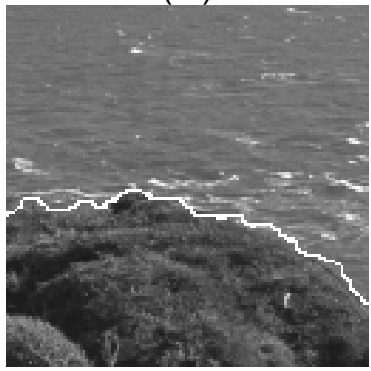

(q)

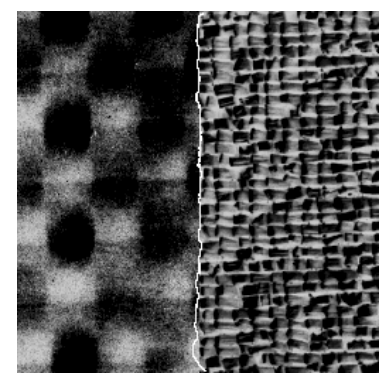

(b)

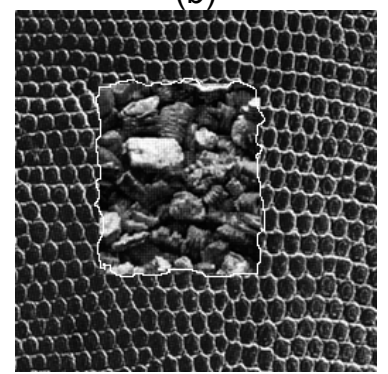

(f)

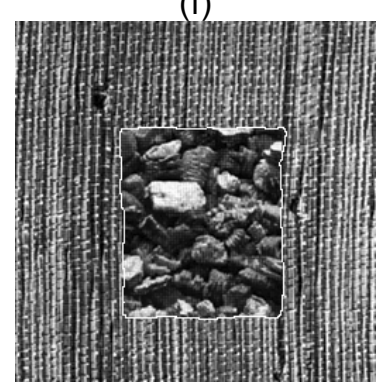

(j)

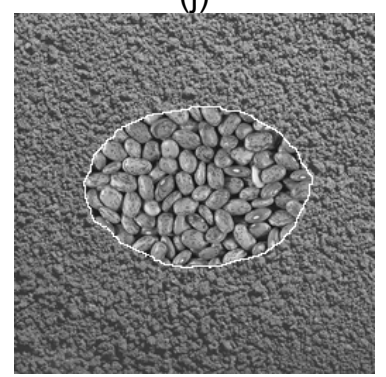

(n)

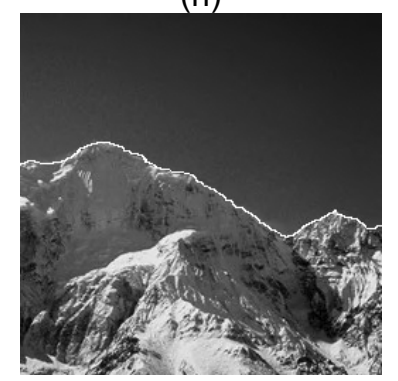

(r)

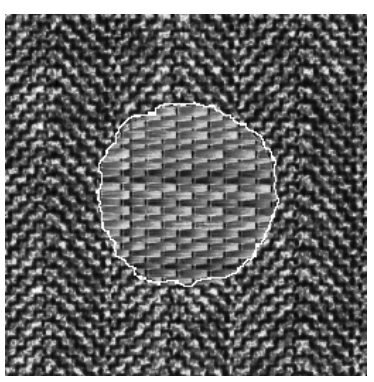

(c)

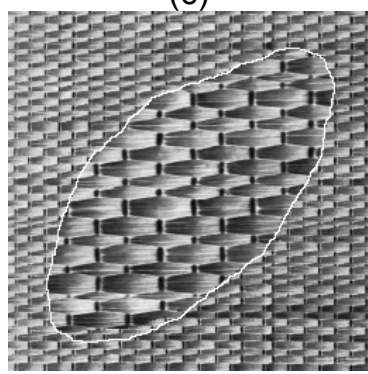

(g)

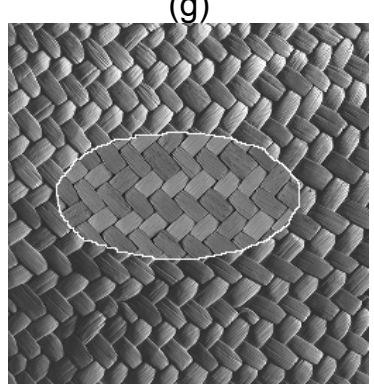

(k)

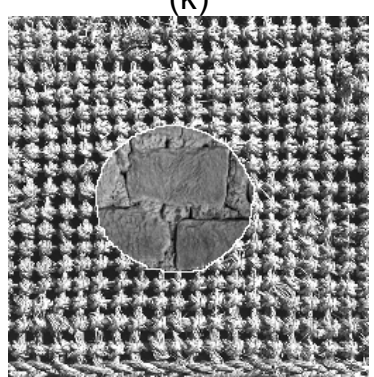

(o)

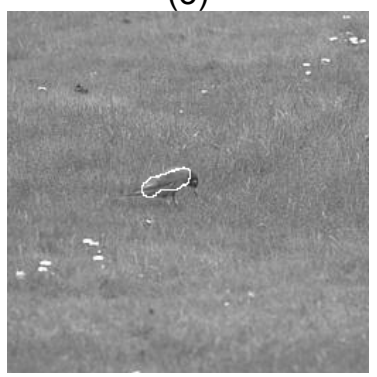

(s)

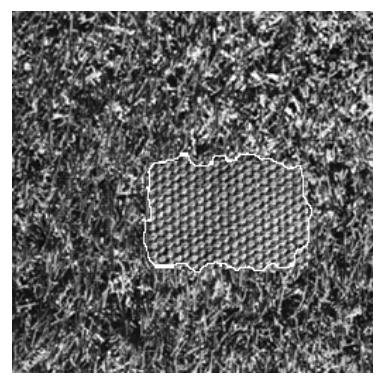

(d)

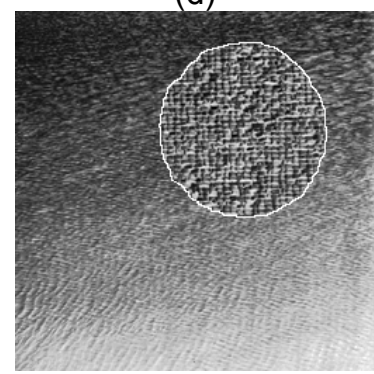

(h)

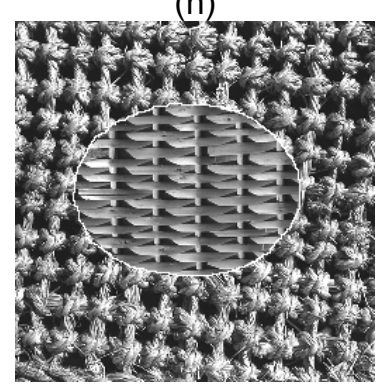

(I)

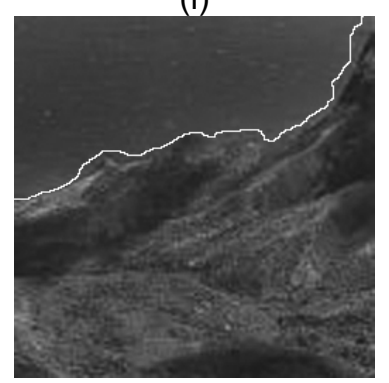

(p)

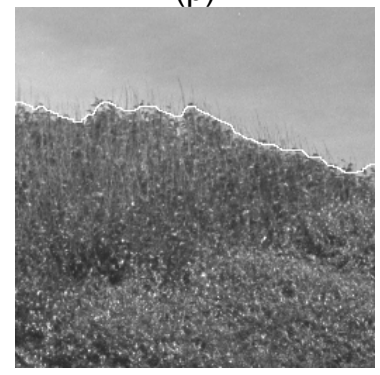

(t)

Fig. 5. Segmentation of the images illustrated in Fig. 3 using the $v$-LAC algorithm. 
As a baseline to compare the segmentation quality obtained by $s$-LAC and $v$-LAC we have considered the JSEG image segmentation algorithm (Deng and Manjunath, 2001). JSEG was applied on each composite image of the available dataset, for multiple combinations of its parameters (scales 1-4; quantization threshold 0-600; and region merging threshold 0.0-1.0). The highest overlaps achieved for each composite image are presented in Table 3 and can be considered as a means to evaluate the difficulty to segment these images. It can be observed that both $s$-LAC and $v$-LAC obtain higher overlaps than JSEG. Moreover, JSEG obtains lower overlaps in images which contain nonstationary textures, as it is the case with Fig. 3f, $3 \mathrm{~h}$ and $3 \mathrm{j}$, indicating that these images pose the most challenging segmentation tasks.

Table 3

Overlaps obtained by a baseline algorithm (JSEG) for the segmentation of the images illustrated in Fig. 3.

\begin{tabular}{cccccc}
\hline Image & Overlap (\%) & Image & Overlap (\%) & Image & Overlap (\%) \\
& & & & & \\
\hline a & 72.3 & $\mathrm{f}$ & 58.1 & $\mathrm{k}$ & 62.8 \\
\hline $\mathrm{b}$ & 68.7 & $\mathrm{~g}$ & 62.3 & $\mathrm{I}$ & 63.2 \\
\hline $\mathrm{c}$ & 94.2 & $\mathrm{~h}$ & 59.6 & $\mathrm{~m}$ & 64.0 \\
\hline $\mathrm{d}$ & 78.9 & $\mathrm{i}$ & 91.4 & $\mathrm{n}$ & 64.5 \\
\hline $\mathrm{e}$ & 88.7 & $\mathrm{j}$ & 56.8 & $\mathrm{o}$ & 92.1 \\
\hline
\end{tabular}

The segmentations of the composite textures illustrated in Fig. 6, as obtained by GAC and IAC in (Sagiv et al, 2004), have $94.3 \%$ and $98.9 \%$ overlaps respectively (Fig. $6 \mathrm{~b}$ ). For the same images, s-LAC gave $94.5 \%$ and $97.2 \%$ overlaps (Fig. 6c), whereas $v$-LAC gave $96.9 \%$ and $99.1 \%$ overlaps (Fig. 6d). These results indicate that the segmentation quality obtained by $s-L A C$ and $v$-LAC can be comparable or higher than the one obtained by state of the art active contours.
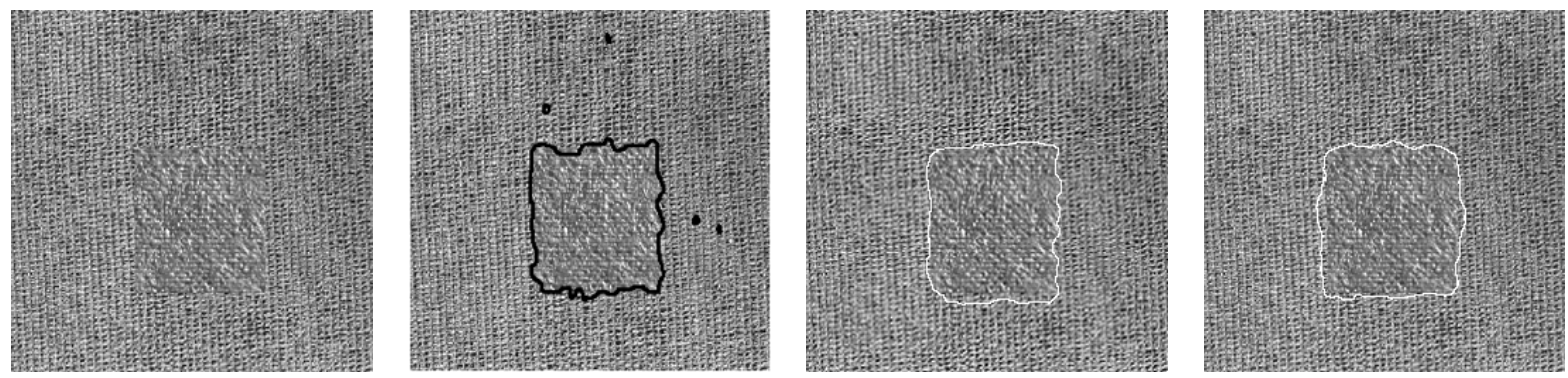


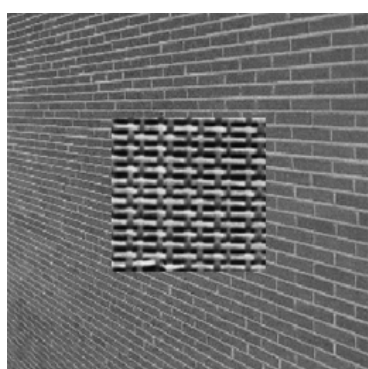

(a)

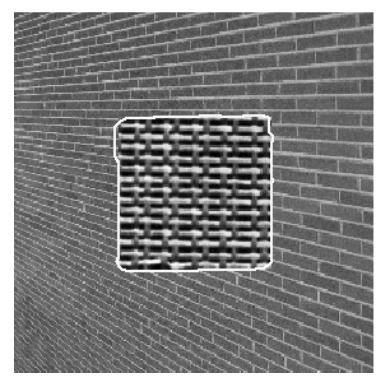

(b)

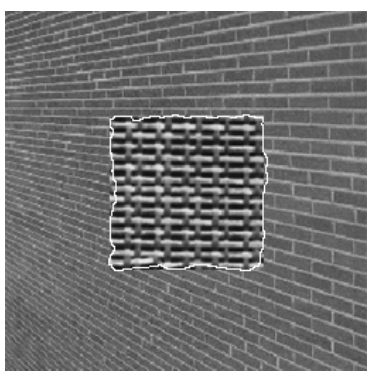

(c)

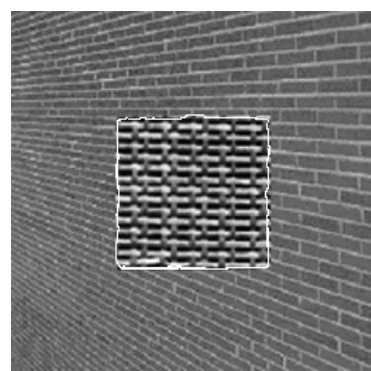

(d)

Fig. 6. (a) Test composite texture images used in (Sagiv et al, 2004), (b) segmentations obtained by GAC (upper image) and IAC (lower image) in (Sagiv et al, 2004), (c) segmentations obtained by s-LAC and (d) segmentations obtained by $v$-LAC.

In the comparative evaluation of five texture segmentation methods included in (Acharyya and Kundu, 2001) the best result reported for the Brodatz D17D55 image (Fig. 3c), was $98.3 \%$ correctly classified pixels. For the same image, s-LAC and $v$-LAC results in $98.7 \%$ and $99.4 \%$ correctly classified pixels, respectively. In another comparative study (Randen and Husoy, 1999), the best classification rate reported among nine different methods was $98.1 \%$ for the Brodatz D4D84 image (Fig. 3a). For the same image, s-LAC and $v$-LAC achieved $99.0 \%$ and $99.8 \%$ respectively.

The overall segmentation time, obtained when s-LAC and $v$-LAC are applied successively, is more than 12 times less than the segmentation times reported on the recent wavelet-based active contour texture segmentation method proposed in (Aujol et al, 2003), whereas Sagiv et al, (2004) do not address the overall segmentation times of IAC. However, in the case of IAC, illumination invariance is obtained by modifying the original equation of ACWE evolution (7) so as to incorporate the inverse of the determinant of the Gabor features submanifold's metric, whereas LBPguided active contours avoid the time consuming calculation of such quantities.

\section{Conclusions}

In this study, we investigated novel approaches to texture segmentation that utilize LBP-based features for the guidance of active contours. Two LBP-guided active contours have been proposed: $s$-LAC and $v$-LAC. Their experimental evaluation on textures and natural scenes, acquired from standard databases, showed that s-LAC enables efficient, high quality texture segmentation by avoiding the iterative calculation of the active contour equation terms derived from LBP feature vectors. Moreover, the experiments showed that $s$-LAC is robust to illumination changes, a capability mainly associated with the LBP texture representation, whereas it allows the segmentation of regions with non-stationary textures. These capabilities are also valid for $v$-LAC, which although computationally more demanding, it can lead to higher segmentation quality than s-LAC, especially if the distance between the LBP feature vectors is measured by means of the log-likelihood statistic. The LBP-guided active contours compete state of the art active contours in texture segmentation. It has been shown that s-LAC can result in a comparable or better segmentation performance than the texture segmentation approaches presented in (Sagiv et al, 2004; Randen and Husoy, 1999; Acharyya and Kundu, 2001), with less computational effort. On the other hand, $v$-LAC can provide 
segmentations of higher quality at the cost of time performance. The iterations of the $v$-LAC algorithm can be reduced by the successive application of $s$-LAC and $v$-LAC, thus requiring less computational effort than the active contours in (Aujol et al, 2003; Sagiv et al, 2004) to perform the segmentation task.

In the experimental evaluation of the proposed LBP-guided active contours, we considered LBPs of a single pixel radius, favoring microtexture segmentation and computational efficiency. However, in case of macrotextures, larger LBP radii could be straightforwardly used instead. Depending on the application, further optimizations of the proposed LBP-guided active contours could also include the utilization of the rotation invariant LBP operator (Ojala et al, 2002), as well as of the multiphase active contour formulation (Vese and Chan, 2002) for the segmentation of multiple textures.

Future perspectives of this work include the formulation of LBP-guided active contours for bimodal segmentation of textures with stationary global minimum (Lee and Seo, 2006), and applications on various domains, including medical and satellite images.

\section{Acknowledgment}

This work was supported by the Greek General Secretariat of Research and Technology and the European Social Fund, through the PENED 2003 program (grant no. 03-ED-662).

\section{References}

Acharyya, M., Kundu, M.K., 2001. An Adaptive Approach to Unsupervised Texture Segmentation Using M-Band Wavelet Transform. Sig. Proc. 81, $1337-1356$.

Akgul, Y.S., Kambhamettu, C., 2003. A Coarse-to-Fine Deformable Contour Optimization Framework. IEEE Trans. on Patt. Anal. Mach. Intell. 25 (2), 174-186.

Allili, M.S., Ziou, D., Bentabet, L., 2004. A Robust Level Set Approach for Image Segmentation and Statistical Modeling. In: Proc. Adv. Conc. Intell. Vis. Syst. (ACIVS), pp. 243-251.

Aubert, G., Vese, L., 1997. A Variational Method in Image Recovery. SIAM J. Num. Anal. 34 (5), 1948-1979.

Aujol, J.F., Aubert, G., Blanc-Feraud, L., 2003. Wavelet-Based Level Set Evolution for Classification of Textured Images. IEEE Trans. Im. Proc. 12 (12), 1634-1641.

Aujol, J.F., Chan, T.F., 2006. Combining Geometrical and Textured Information to Perform Image Classification. J. Vis. Comm. Im. Repr. 17 (5) 1004-1023

Awate S.P., Tasdizen T., Whitaker R.T., 2006. Unsupervised Texture Segmentation with Nonparametric Neighborhood Statistics. In: Proc. Eur. Conf. Comp. Vis. (ECCV), 494-507.

Brodatz, P., 1996. Textures: A Photographic Album for Artists and Designers, New York, NY, Dover.

Caselles, V., Kimmel R., Sapiro, G., 1997. Geodesic Active Contours. Int. J. Comp. Vis. 22, 61-79.

Chan, T., Sandberg, B., Vese, L., 2002. Active Contours Without Edges for Vector-Valued Images. J. Vis. Comm. Im. Repr. 11, $130-141$.

Chan, T.F., Vese, L.A., 2001. Active Contours Without Edges. IEEE Trans. Im. Proc. 7, 266-277.

Deng, Y., Manjunath, B.S., 2001. Unsupervised Segmentation of Color-Texture Regions in Images and Video. IEEE Trans. on Patt. Anal. Mach. Intell. 23 (8), 800-810. 
Duda, R.O., Hart, P.E., Stork, D.G., 2001. Pattern Classification. 2nd edition, Willey.

Feng, X., Pietikäinen, M., Hadid, A., Xie, H., 2005. A Novel Real Time System for Facial Expression Recognition. In: Proc. Aff. Comput. Intell. Inter., Lect. Not. Comp. Sc. 3784, pp. 248-256.

He, Y., Luo, Y., Hu, D., 2004. Unsupervised Texture Segmentation via Applying Geodesic Active Regions to Gaborian Feature Space. IEEE Trans. Eng., Comp. Tech., 272-275.

Hermes, L., Buhmann, J.M., 2003. A Minimum Entropy Approach to Adaptive Image Polygonization. IEEE Trans. Im. Proc. 12 (10), $1243-1258$.

Huang, X., Metaxas, D., Chen, T., 2004. Metamorphs: Deformable Shape and Texture Models. In: Proc IEEE Int. Conf. Comp. Vis. Patt. Rec. (ICVPR), 1496-1503.

Kass, M., Witkin, A., Terzopoulos, D., 1988. Snakes: Active Contour Models. Int. J. Comp. Vis. 1, 321-331.

Kimmel, R., 2003. Geometric Level Set Methods in Imaging, Vision and Graphics. Springer-Verlag.

Lee, S.H., Seo, J.K., 2006. Level Set-Based Bimodal Segmentation with Stationary Global Minimum. IEEE Trans. Im. Proc. 15(9), $2843-2852$.

Lee, S.M., Abott, A.L., Clark, N.A., Araman, P.A., 2005. Active Contours on Statistical Manifolds and Texture Segmentation. In: Proc. IEEE Int. Conf. Im. Proc. (ICIP) 3, 828-831.

Lehmann, T., Bredno, J., Spitzer, K., 2001. Texture-Adaptive Active Contour Models. In: Proc. Int. Conf. Adv Patt. Rec., Vol. 2013, 387-396.

Li, J., Wang, J.Z., 2003. Automatic linguistic indexing of pictures by a statistical modeling approach. IEEE Trans. Patt. Anal. Mach. Intell. 25 (9), 1075-1088.

Liapis, S., Sifakis, E., Tziritas, G., 2004. Colour and Texture Segmentation Using Wavelet Frame Analysis, Deterministic Relaxation, and Fast Marching Algorithms. J. Vis. Comm. Im. Repr. 15, 1-26.

Lucieer, A., Stein, A., 2005. Texture-Based Landform Segmentation of LiDAR Imagery. Int. J. Appl. Earth Obs. Geoinf. 6, 261-270.

Mäenpää, T., 2003. The Local Binary Pattern Approach to Texture Analysis- Extensions and Applications," PhD dissertation, Dept. of Elec. Inf. Eng., Oulou University, Finland.

Mäenpää, T., Pietikäinen, M., 2004. Classification with color and texture: Jointly or separately? Patt. Rec. 37 (8), 1629-1640.

Mäenpää, T., Viertola J., Pietikäinen, M., 2003. Optimising Colour and Texture Features for Real-Time Visual Inspection. Patt. Anal. Appl. 6 (3), 169-175.

Martin, D., Fowlkes, C., Malik, J., 2001. A database of human segmented natural images and its application to evaluating segmentation algorithms and measuring ecological statistics. In: Proc. Int. Conf. Comp. Vis. (ICCV), 416-425.

Mumford, D., Shah, J., 1989. Optimal Approximation by Piecewise Smooth Functions and Associated Variational Problems. Comm. Pur. Appl. Math. 42, 577-685.

Ojala, T., Pietikäinen, M., 1999. Unsupervised Texture Segmentation Using Feature Distributions. Pat. Rec. 32 (3), 477-486.

Ojala, T., Pietikäinen, M., Harwood, D., 1996. A Comparative Study of Texture Measures with Classification based on Feature Distributions," Pat. Rec. 29, 51-59.

Ojala, T., Pietikäinen, M., Mäenpää, T., 2002. Multiresolution Gray-Scale and Rotation Invariant Texture Classification with Local Binary Patterns. IEEE Trans. Patt. Anal. Mach. Intell. 24 (7), 971-987.

Osher S., Sethian, J., 1988. Fronts Propagating with Curvature- Dependent Speed: Algorithms Based on the Hamilton-Jacobi Formulations. J. Comp. Phys. 79, 12-49.

Paclic, P., Duin, R., Kempen, G.V., Kohlus, R., 2002. Supervised Segmentation of Textures in Backscatter Images. In: Proc. IEEE Int. Conf. on Patt. Rec. (ICPR) 2, pp. 490-493.

Paragios, N., 2000. Geodesic Active Regions and Level Set Methods: Contributions and Applications in Artificial Vision. PhD dissertation, Sch. of Comp. Eng., Univ. of Nice, Sophia, Antipolis, France. 
Paragios, N., Deriche, R., 1999. Geodesic Active Contours for Supervised Texture Segmentation. In: Proc. IEEE Int. Conf. on Com. Vis. Patt. Rec., pp. 2422-2427.

Pichler, O., Teuner, A., Hosticka, B.J., 1996. A Comparison of Texture Feature Extraction Using Adaptive Gabor Filtering, Pyramidal and Structured Wavelet Transforms. Patt. Rec. 29 (5), 733-742.

Pujol, O., Radeva, P., 2004. Texture Segmentation by Statistic Deformable Models. Int. J. Im. Graph. 4 (3) 433-452.

Qing, X., 2005. Texture Segmentation Using LBP Embedded Region Competition. El. Lett. Comp. Vis. Im. Anal. 5 (1), 41-47.

Randen, T., Husøy, J.H., 1999. Filtering for Texture Classification: A Comparative Study. IEEE Trans. Patt. Anal. Mach. Intell. 21 (4), 291-310.

Rousson, M., Brox, T., Deriche, R., 2003. Active Unsupervised Texture Segmentation on a Diffusion Based Feature Space. In: Proc. IEEE Int. Conf. Comp. Vis. Patt. Rec., Madison, Wisconsin, USA.

Sagiv, C., Sochen, N.A., Zeevi, Y., 2004. Integrated Active Contours for Texture Segmentation. IEEE Trans. Im. Proc. 1 (1), 1-19.

Sandberg, B., Chan, T.F., 2005. A Logic Framework for Active Contours on Multi-Channel Images. J. Vis. Comm. Im. Repr. 16, 333-358.

Sandberg, B., Chan, T., Vese, L., 2002. A Level-Set and Gabor-based Active Contour Algorithm for Segmenting Textured Images. In: Technical Report 39, Math. Dept. UCLA, Los Angeles, USA.

Sochen, N., Kimmel, R., Malladi, R., 1998. A General Framework for Low Level Vision. IEEE Trans. Im. Proc. 7, 310-318.

Suri, J.S., Liu, K., Laxminarayan, S.N., Zeng, X., Reden, L, 2002. Shape Recovery Algorithms Using Level Sets in 2-D/3-D Medical Imagery: A State-of-the-Art Review. IEEE Trans. Inf. Tech. Biom. 6 (1), 8-28.

Theodoridis, S., Koutroumbas, K., 2006. Pattern Recognition, 3rd edition, Academic Press.

Unser M., Eden, M., 1990. Nonlinear Operators for Improving Texture Segmentation Based on Features Extracted by Spatial Filtering. IEEE Trans. Syst., Man .Cyber. 20 (4), 804-815.

Vese, L.A., Chan, T.F., 2002. A Multiphase Level Set Framework for Image Segmentation Using the Mumford and Shah Model. Int. J. Comp. Vis. 50 (3), 271-293.

Vision Texture Database, MIT Media Lab, www-white.media.mit.edu/vismod/imagery/ VisionTexture/vistex.html.

Xu, C., Prince, J.L., 1998. Generalized Gradient Vector Flow External Forces for Active Contours. Sig. Proc. 71, 131-139.

Zhang, H., Gao, W., Chen, X., Zhao, D., 2006.Object Detection Using Spatial Histogram Features. Im. Vis. Comp. 24, 327-341. 